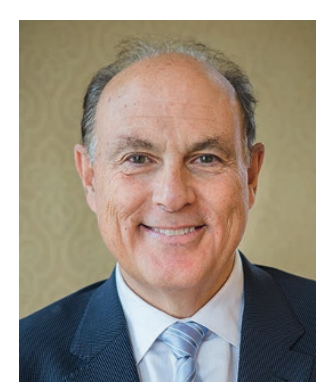

\title{
Reducing violence as the next great public health achievement
}

\author{
Violence is the greatest public health problem of our time, but is the only epidemic in \\ which the health sector is not leading the response, says Gary Slutkin.
}

M ore than 1 billion children - half of all children in the world - are exposed to violence every year. Over 1 million children are estimated to be killed every year from violence of all forms. Over the last few years, we have seen surges in violent behaviour - wars in the Middle East, shootings in cities in the Americas, and mass killings in Paris, Istanbul, Baghdad, Brussels, Orlando and elsewhere. The innumerable shootings and bombings around the globe make it seem like the world is spiralling out of control. This is what it feels like to be inside an epidemic.

Violence is a major global problem due to the death and injury it causes and the profound effects of trauma on individuals, communities and countries. It tears up the physical, psychological, social and economic health of all residents in affected areas. It reduces life expectancy and blocks opportunity. Violence is estimated to cost the world US\$10-14 trillion each year.

It is urgent that we change course and address violence as a health contagion - by interrupting the spread, changing behaviours and shifting norms through well-proven public health outreach and epidemic control strategies.

Primitive thinking about persons committing violence is that they are 'bad' or making 'bad choices'. These ideas are scientifically ungrounded. In fact, we've seen this moralistic thinking repeatedly in the history of science and medicine. During the Middle Ages, persons with plague, leprosy or other contagious diseases, mental illnesses or seizures were regarded as having moral failures, which led to highly unsuccessful and harmful treatment - as we see today with violence.

To say violence is a contagious brain process is not a metaphor, but based on scientific evidence. Society has known for over 50 years that most acquisition of behaviour is through social learning. This unconscious copying means behaviour is transmitted from person to person (that is, it is contagious). Considering different behaviours, violence is particularly contagious due to its salience and traumatic brain effects.
For all other contagious health problems - such as HIV/AIDS, influenza or Ebola - when outbreaks spread, they are correctly identified as epidemics and public health methods to control and eradicate epidemics are rapidly deployed. With recent Ebola outbreaks, the world rallied to collect resources and develop a sophisticated public health response. However, a similar public health response and resource deployment is not occurring with epidemic violence because the problem is still fundamentally misdiagnosed. We have been negligently slow to recognize violence as a contagious health issue.

The health sectors have an impressive global record of effective prevention, changing behaviours and shifting norms, even with the most difficult-to-reach populations. Public health approaches are effective at changing even deep and longstanding behaviours such as unhealthy sexual behaviour or family burial practices. Considering violent behaviour, the health sector must be deployed in a similar way to identify individuals, groups and organizations who may become or are violent, reach them through people they deeply trust, help them deal with whatever drives their unhealthy behaviour, and change the social and other pressures.

This approach should be used for all syndromes of violence from street violence to family violence to war.

Many communities have already deployed these powerful public health methods against violence with great success. For example, New York City has implemented a network of violence intervention and support systems operating across 17 communities, led by the public health sector. The foundation of this network is Cure Violence, a nongovernmental organization that employs the evidence-based public health approach to reduce violence, and that is working in more than 50 communities around the United States and on four continents.

Fifteen years of communitybased practice and evaluations from Chicago to Honduras to South Africa have demonstrated that systematic health-community partnerships that employ epidemic control methods reduce violence, in some cases by $70 \%$ or more (for example, five communities in San Pedro Sula, Honduras showed an $88 \%$ decrease in shootings and killings over two years, using the Cure Violence epidemic control model). Some communities that were heavily affected by violence, such as neighborhoods in Baltimore, New Orleans, New York City and Yonkers have gone for up to two years without a single event of lethal violence.

The epidemic control method for reducing violence - highly recognizable to those who have participated in other infectious disease and epidemic control activities - utilizes trained, supervised and supported specialized community-based health workers who map out areas with the highest incidence of transmission of symptoms or 'disease', reach out to those who have early or more developed signs, reduce the likelihood of further events, and detect and treat close contacts and others at next highest risk. This works for violence as it works for AIDS, tuberculosis or Ebola.

Just as we know that it is more effective and cost-efficient to treat drug addiction as a health issue than to punish those addicted, likewise, it makes more sense to prevent violent events and provide treatment through methods that change behaviours and norms. Like all serious and harmful behaviours for example, drug addiction, smoking and risky sexual behaviour - violence should be understood through this health lens. Public health methods have helped to expand the human lifespan by decades, greatly reduced infant and child deaths, and put many of the great diseases and killers of humankind into the past. Using a science-based epidemic control approach, violence can be reduced to fewer and fewer events, and this will be the next great achievement of science and public health. The sooner the better.

Gary Slutkin is the founder and CEO of Cure Violence, and professor of Epidemiology and Global Health at the University of Illinois, $1603 \mathrm{~W}$. Taylor Street, Chicago, Illinois 60612, USA.

e-mail: gslutkin@uic.edu 